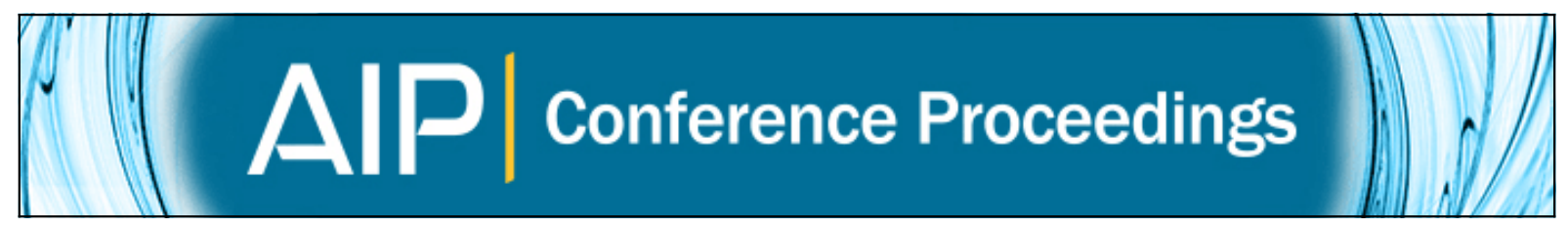

\title{
Two critical issues in Langevin simulation of gas flows
}

Jun Zhang and Jing Fan

Citation: AIP Conference Proceedings 1628, 1087 (2014); doi: 10.1063/1.4902714

View online: http://dx.doi.org/10.1063/1.4902714

View Table of Contents: http://scitation.aip.org/content/aip/proceeding/aipcp/1628?ver=pdfcov

Published by the AIP Publishing

\section{Articles you may be interested in}

Flow field simulation of gas-water two phase flow in annular channel

AIP Conf. Proc. 1592, 130 (2014); 10.1063/1.4872096

Theoretical fundamentals, critical issues, and adequate formulation of effective shale gas and condensate reservoir simulation

AIP Conf. Proc. 1453, 155 (2012); 10.1063/1.4711168

Simulation of Gas Water Two-phase Flow in Diesel Turbocharger

AIP Conf. Proc. 1207, 723 (2010); 10.1063/1.3366453

PDF model based on Langevin equation for polydispersed two-phase flows applied to a bluff-body gas-solid flow Phys. Fluids 16, 2419 (2004); 10.1063/1.1718972

The simulation of spinodal decomposition in two dimensions: A comparison of Monte Carlo and Langevin dynamics

J. Chem. Phys. 79, 1948 (1983); 10.1063/1.445975 


\title{
Two Critical Issues in Langevin Simulation of Gas Flows
}

\author{
Jun Zhang ${ }^{\mathrm{a}, \mathrm{b}}$ and Jing Fan ${ }^{\mathrm{b}}$ \\ ${ }^{a}$ James Weir Fluids Laboratory, Department of Mechanical and Aerospace Engineering, \\ University of Strathclyde, Glasgow G1 1XJ, United Kingdom \\ ${ }^{b}$ State Key Laboratory of High Temperature Gas Dynamics, Institute of Mechanics, \\ Chinese Academy of Sciences, Beijing 100190, China
}

\begin{abstract}
A stochastic algorithm based on the Langevin equation has been recently proposed to simulate rarefied gas flows. Compared with the direct simulation Monte Carlo (DSMC) method, the Langevin method is more efficient in simulating small Knudsen number flows. While it is well-known that the cell sizes and time steps should be smaller than the mean free path and the mean collision time, respectively, in DSMC simulations, the Langevin equation uses a drift term and a diffusion term to describe molecule movements, so no direct molecular collisions have to be modeled. This enables the Langevin simulation to proceed with a much larger time step than that in the DSMC method. Two critical issues in Langevin simulation are addressed in this paper. The first issue is how to reproduce the transport properties as that described by kinetic theory. Transport coefficients predicted by Langevin equation are obtained by using GreenKubo formulae. The second issue is numerical scheme with boundary conditions. We present two schemes corresponding to small time step and large time step, respectively. For small time step, the scheme is similar to DSMC method as the update of positions and velocities are uncoupled; for large time step, we present an analytical solution of the hitting time, which is the crucial factor for accurate simulation. Velocity-Couette flow, thermal-Couette flow, Rayleigh-Bénard flow and wall-confined problem are simulated by using these two schemes. Our study shows that Langevin simulation is a promising tool to investigate small Knudsen number flows.
\end{abstract}

Keywords: Langevin dynamics, Fokker-Planck equation, Transport properties, DSMC method PACS: 47.45.Ab, 51.10.+y, 47.11.Mn

\section{INTRODUCTION}

Direct simulation Monte Carlo (DSMC) method [1] has been very popular in simulating rarefied gas flows since it was proposed by Bird in 1960s. The applications include aerospace engineering, vacuum engineering, micro/nanoscale gas flows, etc. The fundamental idea of DSMC method is the uncoupling of molecular movements and intermolecular collisions within small time intervals. In each calculating time step, the positions of molecules are updated according to their velocities, and then the velocities of molecules are updated for each collision pairs in each collision cells. It has been shown that the numerical transport coefficients are dependent on the cell sizes and time steps due to the uncoupling approximation [2,3]. In order to obtain simulation results correctly, the cell sizes should be less than mean free path, and the time steps should be less than mean collision time. These limitations make the simulation for small Knudsen number flows using DSMC method become very expensive. Many efforts have been made to break the limitations. One promising method is Langevin dynamics.

In Langevin dynamics, two force terms, i.e., a drag force and a random force are employed in the Newton's second law to describe a continuous stochastic process. The details of inter-molecular collisions don't need to be considered. In one calculating time step, one molecule may have experienced several collisions. In this way, the cells sizes and time steps in Langevin simulation are free from the limitations of mean free path and mean collision time. According to the stochastic process theory, Langevin simulation could be considered as a particle method of solution of Fokker-Planck equation [4], which has been proven to be an approximation of Boltzmann equation in moderate or small Knudsen number conditions.

Two critical issues about Langevin simulation are discussed in this paper. The first issue is related to transport properties. The transport coefficients including diffusion, viscosity and thermal conductivity coefficients are evaluated by using Green-Kubo formulae for Langevin model, including velocity model and acceleration model. The second issue is numerical scheme. In order to deal with boundary conditions properly, two situations are considered. For the small time step, uncoupled scheme could be employed and hence linear interpolation could be used to determine hitting time; for the large time step, we obtain analytical solution of hitting time based on theory of Wiener process, and then an efficient scheme of Langevin simulation with large time step is proposed.

Proceedings of the 29th International Symposium on Rarefied Gas Dynamics AIP Conf. Proc. 1628, 1087-1094 (2014); doi: 10.1063/1.4902714

(C) 2014 AIP Publishing LLC 978-0-7354-1265-1/\$30.00 


\section{TRANSPORT PROPERTIES}

In the field of fluid mechanics, transport properties are the most important issues to describe fluid flows correctly. Many simulation methods are criticized because they can't predict transport properties in consist with experimental values. In this paper, the transport coefficients determined by Langevin model are obtained by using Green-Kubo relations. The Green-Kubo relations give the exact mathematical formulae for transport coefficients in terms of integrals of time correlation functions of some specific microscopic flux. Based on the Langevin model and Ito calculus, we could directly obtain the analytical solution of velocity for each molecule, and then the time correlation functions are determined. Using Green-Kubo formula, we obtain analytical solutions of transport coefficients for the Langevin velocity model and acceleration model, respectively [5].

\section{Langevin Velocity Model}

Let us consider simple Langevin model for velocity $u$ of one molecule in $x$ direction,

$$
\frac{d u}{d t}=-\frac{1}{\tau}(u-U)+\left(\frac{4 e_{s}}{3 \tau}\right)^{1 / 2} \frac{d w}{d t},
$$

where $U$ is the mean, or macroscopic velocity of molecules, $\tau$ is the characteristic relaxation time scale of molecular velocities, $e_{s}$ refers to the specific kinetic energy of molecules, i.e., $e_{s}=\frac{3}{2}<u^{2}>$, and $w(\mathrm{t})$ is a Wiener process with the following properties

$$
\begin{aligned}
\left\langle\frac{d w}{d t}(t)\right\rangle & =0, \\
\left\langle\frac{d w}{d t}(t) \frac{d w}{d t}\left(t^{\prime}\right)\right\rangle & =\delta\left(t-t^{\prime}\right) .
\end{aligned}
$$

Note that our analysis is performed under equilibrium condition, and hence $U$ equals to zero. Meanwhile, the kinetic energy is constant and equals to $\frac{3}{2} R T$, where $R$ is the gas constant, and $T$ is the temperature of system.

Using Ito calculus, the exact solution of Eq. (1) could be written as

$$
u(t)=u(0) e^{-\frac{t}{\tau}}+\left(\frac{4 e_{s}}{3 \tau}\right)^{\frac{1}{2}} \int_{0}^{t} e^{-\frac{t-s_{x}}{\tau}} \frac{d w}{d s_{x}}\left(s_{x}\right) d s_{x} .
$$

\section{Diffusion Coefficient}

By taking the average of $u_{x}(0)$ multiplied by $u_{x}(\mathrm{t})$ as Eq. (4), we could obtain

$$
<u(0) u(t)>=<u^{2}(0)>e^{-\frac{t}{\tau}}+\left(\frac{4 e_{s}}{3 \tau}\right)^{\frac{1}{2}}<u(0) \int_{0}^{t} e^{-\frac{t-s_{x}}{\tau}} \frac{d w}{d s_{x}}\left(s_{x}\right) d s_{x}>.
$$

Because $\frac{d w}{d s_{x}}$ is uncorrelated to $u(0)$, the ensemble average makes the last term in Eq. (5) is zero. The time correlation function of velocity is simplified as

$$
<u(0) u(t)>=<u^{2}(0)>e^{-\frac{t}{\tau}}=\frac{2 e_{s}}{3} e^{-\frac{t}{\tau}} .
$$

Consequently, the diffusion coefficient could be obtained using Green-Kubo formula,

$$
D=\int_{0}^{+\infty}<u(0) u(t)>d t=\frac{2 e_{s}}{3} \int_{0}^{+\infty} e^{-\frac{t}{\tau}} d t=\frac{2 e_{s}}{3} \tau .
$$




\section{Viscosity Coefficient}

Due to the independence of particles in Langevin dynamics, the ensemble average terms of inter-particles equal to zero. And thus the time correlation function of microscopic stress can be simplified as

$$
\begin{aligned}
<P_{x y}(0) P_{x y}(t)> & =<\sum_{i=1}^{N} m u_{i}(0) v_{i}(0) \cdot \sum_{j=1}^{N} m u_{j}(t) v_{j}(t)> \\
& =N m^{2}<u(0) v(0) u(t) v(t)>
\end{aligned}
$$

Substituting the solutions for velocities into Eq. (8), we obtain

$$
\begin{aligned}
<P_{x y}(0) P_{x y}( & t)>=N m^{2} e^{-\frac{2 t}{\tau}}<u^{2}(0) v^{2}(0)> \\
+ & N m^{2}\left(\frac{4 e_{s}}{3 \tau}\right)^{\frac{1}{2}}<u(0) v^{2}(0) \int_{0}^{t} e^{-\frac{t-s_{x}}{\tau}} \frac{d w}{d s_{x}}\left(s_{x}\right) d s_{x}> \\
+ & N m^{2}\left(\frac{4 e_{s}}{3 \tau}\right)^{\frac{1}{2}}<u^{2}(0) v(0) \int_{0}^{t} e^{-\frac{t-s_{y}}{\tau}} \frac{d w}{d s_{y}}\left(s_{y}\right) d s_{y}> \\
+ & N m^{2} \frac{4 e_{s}}{3 \tau}<u(0) v(0) \int_{0}^{t} e^{-\frac{t-s_{x}}{\tau}} \frac{d w}{d s_{x}}\left(s_{x}\right) d s_{x} \int_{0}^{t} e^{-\frac{t-s_{y}}{\tau}} \frac{d w}{d s_{y}}\left(s_{y}\right) d s_{y}>
\end{aligned}
$$

Considering that the inter-particles and inter-directions are uncorrelated, and the Wiener process is uncorrelated to initial velocities, the last three terms in Eq. (9) equal to zero. Hence Eq. (9) reduces to

$$
<P_{x y}(0) P_{x y}(t)>=N m^{2} e^{-\frac{2 t}{\tau}}<u^{2}(0)><v^{2}(0)>=\frac{4 e_{s}^{2}}{9} N m^{2} e^{-\frac{2 t}{\tau}} .
$$

Integrating the time correlation function of microscopic stress, we obtain the viscosity coefficient

$$
\mu=\frac{1}{k_{B} V T} \int_{0}^{+\infty}<P_{x y}(0) P_{x y}(t)>d t=\frac{1}{k_{B} V T} \frac{4 e_{s}^{2}}{9} N m^{2} \int_{0}^{+\infty} e^{-\frac{2 t}{\tau}} d t=\frac{P \tau}{2},
$$

where $V$ is the volume of system, $T$ is the temperature, $P$ is the pressure, i.e., $P=n k_{B} T$, and $n$ is the number density, i.e., $n=N / V$.

\section{Thermal Conductivity Coefficient}

Similarly, considering the independence of particles, the time correlation function of microscopic heat flux reduces to

$$
<J_{x}(0) J_{x}(t)>=N<\left(\frac{1}{2} m \mathbf{u}^{2}(0)-\frac{5}{2} k_{B} T\right) u(0) \cdot\left(\frac{1}{2} m \mathbf{u}^{2}(t)-\frac{5}{2} k_{B} T\right) u(t)>,
$$

where $\mathbf{u}^{2}(0)=u^{2}(0)+v^{2}(0)+w^{2}(0)$ and $\mathbf{u}^{2}(t)=u^{2}(t)+v^{2}(t)+w^{2}(t)$.

On substituting solutions for velocities into Eq. (12) and making some simplifications, we obtain

$$
<J_{x}(0) J_{x}(t)>=N<\left(\frac{1}{2} m \mathbf{u}^{2}(0)-\frac{5}{2} k_{B} T\right)^{2} u^{2}(0) e^{-\frac{3 t}{\tau}}>.
$$

By taking the moments of the Maxwellian distribution function under equilibrium condition, we could obtain $<u^{2}(0)>=\frac{2 e_{s}}{3},<u^{4}(0)>=\frac{4 e_{s}^{2}}{3}$ and $\left\langle u^{6}(0)>=\frac{40 e_{s}^{3}}{9}\right.$. And thus Eq. (13) could be written

$$
<J_{x}(0) J_{x}(t)>=\frac{20 e_{s}^{3}}{27} N m^{2} e^{-\frac{3 t}{\tau}} \text {. }
$$

Integrating the time correlation function of microscopic heat flux, we obtain the thermal conductivity coefficient

$$
\kappa=\frac{1}{k_{B} V T^{2}} \int_{0}^{+\infty}<J_{x}(0) J_{x}(t)>d t=\frac{1}{k_{B} V T^{2}} \frac{20 e_{s}^{3}}{27} N m^{2} \int_{0}^{+\infty} e^{-\frac{3 t}{\tau}} d t=\frac{5 P R \tau}{6} .
$$




\section{Prandtl Number}

The Prandtl number is a dimensionless number approximating the ratio of momentum diffusivity and thermal diffusivity and can be expressed as

$$
\operatorname{Pr}=\frac{C_{p} \mu}{\kappa},
$$

where $C_{p}$ is the specific heat capacity. For monatomic gas, $C_{p}=\frac{5}{2} R$. On substituting Eqs. (11) and (15) into

Eq. (16), the Prandtl number equals to $3 / 2$ for monatomic gas. This analytical result is the same as that presented in Jenny et al.'s paper [6], where they derived the Prandtl number by taking moments of the Fokker-Planck equation. It is known that the Prandtl number is $2 / 3$ for monatomic gas, so the simple Langevin velocity model could not predict correct Prandtl number.

\section{Langevin Acceleration Model}

Since the simple Langevin velocity model can not predict correct Prandtl number for monatomic gas, some developments have been made for adjusting Prandtl number [7, 8]. Heinz [7] proposed acceleration model with an additional time scale $\tau_{a}$ for describing molecular movements,

$$
\begin{gathered}
\frac{d u}{d t}=a, \\
\frac{d a}{d t}=\frac{1}{\tau_{a}}\left[-\left(1+\frac{\tau_{a}}{\tau}\right) a-\frac{1}{\tau} u+\left(\frac{4 e_{s}}{3 \tau}\left(1+\frac{\tau_{a}}{\tau}\right)\right)^{1 / 2} \frac{d w}{d t}\right],
\end{gathered}
$$

where $a$ is the acceleration in x direction. The velocity model as Eq. (1) can be recovered by multiplying Eq. (18) by $\tau_{a}$ and taking the limit $\tau_{a} \rightarrow 0$. For the sake of derivation easily, letting $r_{1}=-\frac{1}{\tau}$ and $r_{2}=-\frac{1}{\tau_{a}}$, the solutions for velocity and acceleration could be written as simple form

$$
\begin{aligned}
u(t) & =a(0) \frac{e^{r_{2} t}-e^{r_{1} t}}{r_{2}-r_{1}}+u(0) \frac{r_{2} e^{r_{1} t}-r_{1} e^{r_{2} t}}{r_{2}-r_{1}} \\
& +\frac{1}{r_{2}-r_{1}}\left(-\frac{4 e}{3} r_{1} r_{2}\left(r_{1}+r_{2}\right)\right)^{1 / 2} \int_{0}^{t}\left(e^{r_{2}(t-s)}-e^{r_{1}(t-s)}\right) \frac{d w}{d s}(s) d s
\end{aligned}
$$

By differentiating Eq. (19) with respect to $t$, the expression of acceleration follows as

$$
\begin{aligned}
a(t) & =a(0) \frac{r_{2} e^{r_{2} t}-r_{1} e^{r_{1} t}}{r_{2}-r_{1}}+u(0) \frac{r_{1} r_{2}\left(e^{r_{1} t}-e^{r_{2} t}\right)}{r_{2}-r_{1}} \\
& +\frac{1}{r_{2}-r_{1}}\left(-\frac{4 e}{3} r_{1} r_{2}\left(r_{1}+r_{2}\right)\right)^{1 / 2} \int_{0}^{t}\left(r_{2} e^{r_{2}(t-s)}-r_{1} e^{r_{1}(t-s)}\right) \frac{d w}{d s}(s) d s
\end{aligned}
$$

The velocities and accelerations in y and $\mathrm{z}$ directions have the same forms as Eqs. (19) and (20), respectively. Considering that energy is conserved under equilibrium condition, these relations $<u^{2}(t)>=\frac{2 e_{s}}{3},<a^{2}(t)>=\frac{2 e_{s}}{3} r_{1} r_{2}$ and $\langle u(t) a(t)\rangle=0$ are necessarily satisfied. Using these expressions, the transport coefficients could be obtained by employing Green-Kubo formulae. Here we just present the results, and the details for derivation could be found in our recent paper [5].

$$
\begin{gathered}
D=\frac{2 e_{s}}{3}\left(\tau+\tau_{a}\right), \\
\mu=\frac{p\left(\tau^{2}+3 \tau \tau_{a}+\tau_{a}^{2}\right)}{2\left(\tau+\tau_{a}\right)}, \\
\kappa=\frac{5 p R}{6} \frac{\left(\tau+\tau_{a}\right)\left(2 \tau_{a}^{2}+9 \tau_{a} \tau+2 \tau^{2}\right)}{\left(2 \tau_{a}+\tau\right)\left(\tau_{a}+2 \tau\right)},
\end{gathered}
$$




$$
\operatorname{Pr}=\frac{3\left(2 \tau_{a}+\tau\right)\left(\tau_{a}+2 \tau\right)\left(\tau^{2}+3 \tau \tau_{a}+\tau_{a}^{2}\right)}{2\left(\tau+\tau_{a}\right)^{2}\left(2 \tau_{a}^{2}+9 \tau_{a} \tau+2 \tau^{2}\right)} .
$$

It is obvious that the Prandtl number varies with the ratio of two time scales, i.e., $\tau_{a} / \tau$. When $\tau_{a} / \tau \rightarrow 0$ or $\tau_{a} / \tau \rightarrow+\infty$, the Prandtl number has the maximum value 1.5; when the two time scales are equal, the Prandtl number has the minimum value, which is about 1.298. Comparing with the Langevin velocity model, the Langevin acceleration model could adjust the Prandtl number to some extent, but cannot approach to the correct value $2 / 3$ for monatomic gas. Note that our analysis is performed in the limit of equilibrium, $\tau$ and $\tau_{a}$ are assumed constant. Under non-equilibrium condition, $\tau$ and $\tau_{a}$ may vary in space via temperature dependence, and it is possible to adjust Prandtl number to the correct value $2 / 3$ for monatomic gas.

\section{NUMERICAL SCHEME}

Jenny et al. [6] proposed an efficient algorithm to solve the Langevin equation. In each calculating time step $\Delta t$, the evolution of position and velocity are as follows,

$$
\begin{gathered}
x(\Delta t)=x(0)+U \Delta t+(u(0)-U) \tau\left(1-e^{-\Delta t / \tau}\right)+\sqrt{B} \varepsilon_{1}, \\
u(\Delta t)=u(0)-(u(0)-\mathrm{U})\left(1-e^{-\Delta t / \tau}\right)+\sqrt{\frac{C^{2}}{B}} \varepsilon_{1}+\sqrt{A-\frac{C^{2}}{B}} \varepsilon_{2},
\end{gathered}
$$

where $\varepsilon_{1}$ and $\varepsilon_{2}$ are independent, normal distributed random variables, $A$ and $B$ are the second moments of velocity and displacement, respectively, and $C$ is the joint moment of the velocity and displacement as follow,

$$
\begin{gathered}
A=\frac{2 e_{s}}{3}\left(1-e^{-2 \Delta t / \tau}\right), \\
B=\frac{2 e_{s} \tau^{2}}{3}\left(\frac{2 \Delta t}{\tau}-\left(1-e^{-\Delta t / \tau}\right)\left(3-e^{-\Delta t / \tau}\right)\right), \\
C=\frac{2 e_{s} \tau}{3}\left(1-e^{-\Delta t / \tau}\right)^{2} .
\end{gathered}
$$

Theoretically, the solutions as Eqs. (25) and (26) are accurate for any time steps. However, the solutions are derived without any boundary walls. It is very important to properly employ boundary conditions for simulation of fluid flows. Usually, diffused reflection or Maxwell boundary condition is assumed at boundary walls. The crucial problem is to determine the hitting time. In Jenny et al.'s paper [6], linear interpolation is used to determine the hitting time. However, the movement of molecules as Eq. (25) is not a linear process. Here we consider two limit conditions where hitting time could be obtained accurately.

\section{Small Time Step}

If time step is much smaller than mean collision time, the Eq. (25) could be simplified by using Taylor expansion as follow,

$$
x(\Delta t)=x(0)+u(0) \Delta t+\sqrt{\frac{4 e_{s} \Delta t^{3}}{9 \tau}} \varepsilon_{1} .
$$

Considering that the random part in Eq. (30) is much smaller than the deterministic part, so Eq. (30) could be further simplified as

$$
x(\Delta t)=x(0)+u(0) \Delta t .
$$

It means that the movement in each direction is linear process, and the displacement is proportional to the initial velocity in each calculating time step. In this way, linear interpolation is applicable to determine the hitting time if one molecule hits the boundary wall in one calculating time step. Therefore, the process of molecular movements in this scheme is the same as that in DSMC method. The difference is that the solution as Eq. (26) is used to update the velocities in Langevin simulation with small time step while collision process is considered in DSMC method. The details about this scheme could be found in Gorji and Jenny's paper [9].

Velocity-Couette flow and thermal-Couette flow are simulated by using Langevin simulation with time step $\Delta t=0.1 \tau$. Argon gas molecules at standard conditions are confined between two walls. The Knudsen number 


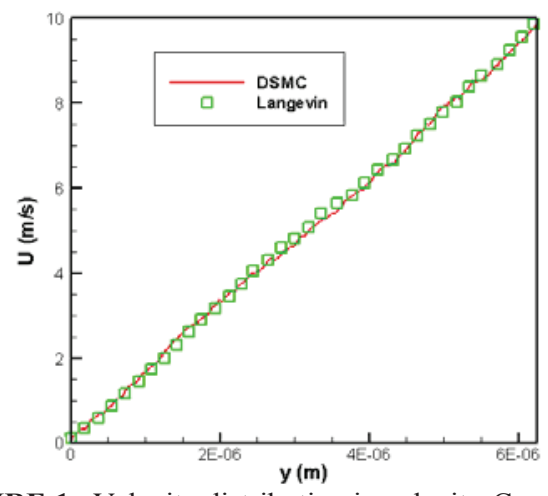

FIGURE 1. Velocity distribution in velocity-Couette flow.

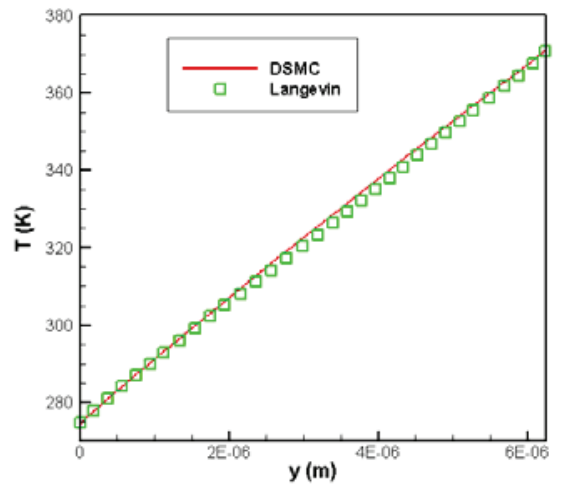

FIGURE 2. Temperature distribution in thermal-Couette flow.

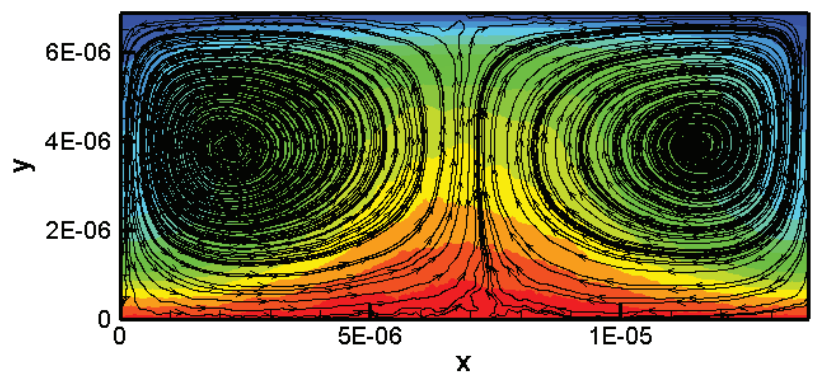

FIGURE 3. Flow patterns obtained by Langevin simulation for $\mathrm{Ra}=4000$.

defined as the ratio of mean free path to the length between two walls is 0.01 . For the sake of comparison, simulation cases are also performed using DSMC method with same time step. As shown in Figs. 1 and 2, the velocity distribution in velocity-Couette flow obtained by Langevin simulation is consistent with that obtained by DSMC method, and the temperature distribution in thermal-Couette flow obtained by two methods are also consistent. As there are no individual collisions in Langevin simulation, the efficient of Langevin simulation is still higher than DSMC method although the time step is as small as that in DSMC method.

We further employ the scheme to simulate two-dimensional Rayleigh-Bénard flow, where molecules are confined between two horizontal parallel walls maintained at different temperatures, and molecules are subjected to external force in vertical direction. Convection vortices will form if the non-dimensional Rayleigh number $(R a)$ exceeds a certain value. Linear stability analysis and simulation results have shown that the critical Ra number is 1708 [10, 11]. Figure. 3 shows the Langevin simulation results for $R a=4000$ and $K n=0.01$, where a pair of counter-rotating vortices are formed in the field.

\section{Large Time Step}

Note that all of the simulations above are performed under small time steps. The main advantage of Langevin simulation comparing with DSMC method is that it could be used with large time step because no direct collisions need to be considered. Here we introduced our recent scheme with large time step. The main progress is the analytical solution of hitting time. If the time step is much larger than mean collision time, the Eq. (25) could be simplified as follow,

$$
x(\Delta t)=x(0)+U \Delta t+(u(0)-U) \tau+\sqrt{\frac{2 e_{s} \tau^{2}}{3}\left(\frac{2 \Delta t}{\tau}-3\right)} \varepsilon_{1} .
$$

Furthermore, we could write the last term in the form of standard Wiener process, 


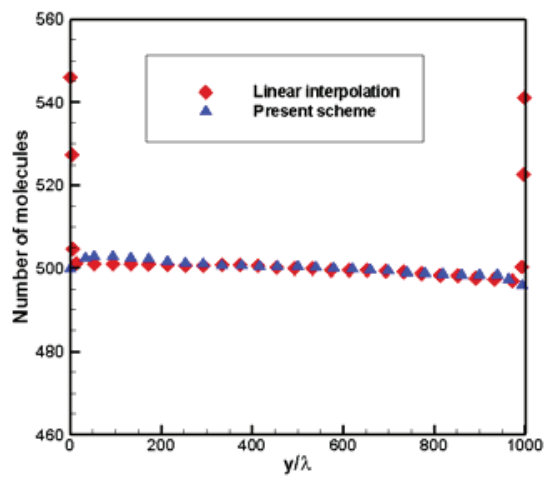

FIGURE 4. Density distribution in one-dimensional wall-confined box.

$$
x(\Delta t)=x(0)+U \Delta t+(u(0)-U) \tau+\sqrt{\frac{4 e_{s} \tau}{3}} W_{\Delta t-3 \tau / 2} .
$$

According to the theory of Wiener process, the hitting time is determined accurately as

$$
\Delta t_{h i t}=\frac{\left|\Delta x_{1}\right|}{\sigma} \sqrt{\frac{\pi T}{2}} \exp \left(\frac{\left(\left|\Delta x_{1}\right|+\left|\Delta x_{2}\right|\right)^{2}}{2 \sigma^{2} T}\right) \operatorname{erfc}\left(\frac{\left|\Delta x_{1}\right|+\left|\Delta x_{2}\right|}{\sqrt{2 \sigma^{2} T}}\right),
$$

where $\Delta x_{1}=x(0)+(u(0)-U) \tau-x_{h i t}, \Delta x_{2}=x(\Delta t)-x_{h i t}, T=\Delta t-3 \tau / 2$ and $\sigma=\sqrt{4 e_{s} \tau / 3}$. Based on the analytical solution of hitting time under the condition of $\Delta t » \tau$, we proposed an efficient scheme with large time step for Langevin simulation. Here we briefly introduce the scheme for one dimensional case. We divide the whole simulation domain into three parts: the bottom part, the central part and the top part. The bottom and top parts are very close to the boundary walls, and the height is less than $4 \lambda$, where $\lambda$ is the mean free path. In each calculating time step, if one molecule hits the boundary in short time period, the scheme with small time step is performed. On the other side, the scheme with large time step could be performed for the molecules in the central part. If one molecule hits the boundary wall, the Eq. (34) is used to determine the hitting time, and then the molecule will move in the remaining time after it gets new velocity at the hitting point. If diffusive reflection is assumed for the boundary wall, the reflecting velocity is randomly selected from a Half-range Maxwellian distribution at the wall temperature.

To validate our scheme with large time step, we perform simulation for Argon gas molecules confined in onedimensional box. The initial state of gas is under standard conditions, i.e., the temperature is $273 \mathrm{~K}$ and the pressure is $1 \mathrm{~atm}$. The length of box is $1000 \lambda$, where $\lambda$ is the mean free path. The wall temperature is fixed at $273 \mathrm{~K}$. Diffusive reflections are assumed at the boundary wall. In order to obtain the distribution of macroscopic quantities, the simulation domain is divided into 300 cells, and each cell is assigned 500 molecules at the initial state. The calculating time step is $10 \tau$, where $\tau$ is the mean collision time of molecules. As shown in Fig. 4, our scheme predicts a uniform density distribution in the field, while the linear interpolation over predicts the density close the boundary walls. Because linear interpolation over predicts the hitting time, and hence the remaining time in one calculating time step becomes smaller. In this way, more molecules will stay close to the boundary walls.

\section{CONCLUSIONS}

In this paper, two critical issues about transport properties and numerical scheme with boundary conditions in Langevin simulation are addressed. The transport coefficients, including diffusion, viscosity and thermal conductivity coefficients predicted by Langevin equation are obtained by using Green-Kubo formulae. For the Langevin velocity model, the time correlation functions have the exact exponent forms, and the predicted Prandtl number is $3 / 2$ for monatomic gas. For the Langevin acceleration model with an additional time scale, the Prandtl number could be adjusted to some extent. We present two numerical schemes. For small time step, the scheme is similar to DSMC method as the update of positions and velocities of molecules are uncoupled. Linear interpolation is proper to determine the hitting time. For large time step, the movements of molecules are no longer linear process, and analytical solution of hitting time could be obtained based on theory of Wiener process. We further propose an efficient scheme for Langevin simulation with large time step, and the accuracy of this scheme is validated in wallconfined problem. Applications of this scheme to more complex gas flows are on-going. 


\section{ACKNOWLEDGMENTS}

The research leading to these results has received funding from the Engineering and Physical Sciences Council (EPSRC) of the UK under grant EP/I011927/1. Part of this work was funded by the National Natural Science Foundation of China (Grant Nos. 11002147 and 11372325). Results were obtained using the ARCHIE-WeSt High Performance Computer (www.archie-west.ac.uk), under EPSRC grant EP/K000586/1. The authors thank Thomas Önskog for his theoretical analysis of numerical scheme and derivation of the hitting time presented in this paper.

\section{REFERENCES}

1. G.A. Bird, Molecular Gas Dynamics and Direct Simulation of Gas Flows, Oxford: Clarendon, 1994.

2. F.J. Alexander, A.L. Garcia and B.J. Alder, Physics of Fluids, 10, 1540-1542 (1998).

3. A.L. Garcia and W. Wagner, Physics of Fluids, 12, 2621-2633 (2000).

4. H. Risken, The Fokker-Planck Equation, Berlin: Springer-Verlag, 1984.

5. J. Zhang, D.D. Zeng and J. Fan, Physica A: Statistical Mechanics and its Applications, 411, 104-112 (2014).

6. P. Jenny, M. Torrilhon and S. Heinz, Journal of Computational Physics, 229, 1077-1098 (2010).

7. S. Heinz, Physical Review E, 70, 036308 (2004).

8. M.H. Gorji, M. Torrilhon and P. Jenny, Journal of Fluid Mechanics, 680, 574-601 (2011).

9. M.H. Gorji and P. Jenny, Journal of Computational Physics, 262, 325-343 (2014).

10. J. Zhang and J. Fan, Physical Review E, 79, 056302 (2009).

11. J. Zhang, J. Fan and F. Fei, Physics of Fluids, 22, 122005 (2010). 